\title{
Multipartite entanglement, quantum coherence and quantum criticality in triangular and Sierpiński fractal lattices
}

\author{
Jun-Qing Cheng and Jing-Bo $\mathrm{Xu}^{*}$ \\ Zhejiang Institute of Modern Physics and Physics Department, Zhejiang University, Hangzhou 310027, China
}

(Dated: June 25, 2021)

\begin{abstract}
We investigate the quantum phase transitions of the transverse-field quantum Ising model on the triangular lattice and Sierpiński fractal lattices by employing the multipartite entanglement and quantum coherence along with the quantum renormalization group method. It is shown that the quantum criticalities of these high-dimensional models closely relate to the behaviors of the multipartite entanglement and quantum coherence. As the thermodynamic limit is approached, the first derivatives of the multipartite entanglement and quantum coherence exhibit singular behaviors, and the consistent finite-size scaling behaviors for each lattice are also obtained from the first derivatives. The multipartite entanglement and quantum coherence are demonstrated to be good indicators for detecting the quantum phase transitions in the triangular lattice and Sierpiński fractal lattices. Furthermore, the dimensions determine the relations between the critical exponents and the correlation length exponents for these lattices.
\end{abstract}

PACS numbers: 05.30.Rt, 03.67.Mn, 75.10.Pq, 64.60.al

\section{INTRODUCTION}

Quantum phase transitions (QPTs) are notable manifestations of quantum many-body systems at absolute zero temperature where the quantum fluctuations play a dominant role and no thermal fluctuations exist [1]. QPTs can be achieved by changing the parameters of Hamiltonian, such as an external magnetic field or the coupling constant. As a control parameter is varied through a critical value, the ground state of a system suffers an abrupt change mapped to a variation in the system's properties. How to reveal and characterize the critical phenomenons of quantum many-body systems is an important task and becomes a hot topic in condensedmatter physics. Traditional methods mainly focus on the identification of the order parameters and the pattern of symmetry breaking. Recent developments in quantum information theory [2] have provided some insights into the QPTs. Specifically, the quantum entanglement has been successfully used as an effective tool to reveal the QPTs without any prior knowledge of the order parameter [3-6]. Since the concept of renormalization was introduced from the quantum field theory to quantum statistical physics [7], many progresses have been made in the research of QPTs. As a variant of renormalization group at zero temperature, quantum renormalization group $(\mathrm{QRG})$ is a tractable method for studying the criticalities of one-dimensional [8-11] and two-dimensional $[12,13]$ many-body systems. This method can be used to evaluate the quantum critical points and scaling behaviors analytically, but has difficulties in quantitative estimation for the transverse-field Ising model [14, 15]. Recently, it has been shown that a novel renormalization group (RG) map can not only be used to accurately examine the critical behavior of the one-dimensional quantum transverse-field Ising model, and also be used to

* Emali: xujb@zju.edu.cn predict the critical behaviors of the higher-dimensional models $[16,17]$. In particular, there have been efforts to study the quantum Ising models on fractal lattices [1719] which were not clear before. Fractals are self-similar structures in noninteger dimensions and have both aesthetic and scientific interests. They have been used to interpolate between integer dimensional regular lattices and construct the networks for quantum computation and communication $[20,21]$. The quantum criticalities of fractal lattices attract our attention.

On the other hand, the entanglement in the ground state of a many-body system can be utilized as a resource for quantum technologies [6]. The multipartite entanglement offers significant advantages in quantum tasks compared with bipartite entanglement. For example, it is the main ingredient in measurement-based quantum computation [22] and various quantum communication protocols [23-25]. Therefore, the entanglement quantification of multipartite quantum states is necessary and essential in quantum information science. The monogamy of entanglement is one of most important properties in manybody quantum systems [26], and can be used to characterize the entanglement structure. It has been recently discovered that the squared entanglement of formation obeys the monogamy inequality in an arbitrary $N$-qubit mixed state, and a relevant multipartite entanglement indicator is proposed [27]. The multipartite entanglement provides a global view and more physical insights into the characters of a many-body system, and it may have some advantages over bipartite entanglement to reveal the QPTs. Furthermore, the quantum coherence, which arises from the quantum superposition principle, plays a very important role in the fields of quantum optics [28] and quantum information [2]. However, there has been no well-accepted efficient method for measuring the quantum coherence until very recently. A rigorous theoretical framework for quantifying the quantum coherence and the necessary constraints for the quantifier have been proposed [29]. It is interesting to do some research about the multipartite entanglement and quantum coherence in 
the QPTs of high-dimensional many-body systems.

These developments on QPTs, QRG method, multipartite entanglement and quantum coherence motivate us to consider the following questions: How do the multipartite entanglement and quantum coherence behave in the QPTs of high-dimensional models? Can the multipartite entanglement and quantum coherence be used to indicate the QPTs of the transverse-field quantum Ising models on the fractal lattices? If we can apply the QRG approach to find the finite-size scaling behaviors proposed in Ref. [4] for the cases of fractal lattices? Are the critical exponents of multipartite entanglement consistent with the ones of quantum coherence for the same lattice? What are the relations between the critical exponents and correlation length exponents for high-dimensional systems? In this paper, we investigate the performances of multipartite entanglement and quantum coherence in the QPTs for transverse-field quantum Ising model on the triangular lattice and Sierpiński fractal lattices by employing the QRG method. It is found that the quantum criticalities of these models closely relate to the behaviors of the multipartite entanglement and quantum coherence. The singularities for each lattice are observed from the first derivatives of the multipartite entanglement and quantum coherence. The scaling behaviors as introduced in Ref. [4] are obtained for these lattices, especially the ones which describe how the critical points are touched as the thermodynamic limit is approached haven't been discussed before. It is also shown that the multipartite entanglement and quantum coherence obey the universal finite-size scaling laws for the same lattice. Furthermore, the dimensions of lattices play the decisive roles on the relations between the critical exponents and correlation length exponents. The multipartite entanglement and quantum coherence are proven to be good indicators to detect the QPTs of the transverse-field quantum Ising model on the high-dimensional lattices, such as the triangular and Sierpiński fractal lattices.

This paper is organized as follows. In Sec. II, we study the QPTs of lattices by employing multipartite entanglement along with the QRG method. In Sec. III, we investigate the quantum coherence and the QPTs of lattices by using the QRG method. Finally, the conclusions are drawn in Sec. IV.

\section{MULTIPARTITE ENTANGLEMENT AND QUANTUM PHASE TRANSITIONS IN LATTICES}

We consider a set of localized spin- $1 / 2$ particles in the triangular lattice or Sierpiński fractal lattices coupled through exchange interaction $J$ and subject to an external magnetic field of strength $h$. The Hamiltonians for such transverse-field quantum Ising models are given by

$$
H=-J \sum_{\langle i, j\rangle} \sigma_{i}^{z} \sigma_{j}^{z}-h \sum_{i} \sigma_{i}^{x}
$$

where $\sigma_{i}^{\alpha}(\alpha=x, z)$ are the standard spin-1/2 Pauli operators at the site $i$. The sums are over all the nearest neighbor pairs and over all sites, respectively. We mainly focus on the ferromagnetic interactions $J>0$ and the transverse field $h \geqslant 0$. In this work, three kinds of lattices as shown in Fig. 1 are considered, which are the triangular lattice and Sierpiński fractal lattices, respectively. For simplicity, the exchange interaction normalized to the transverse field strength $g=J / h$ is applied during our investigation.

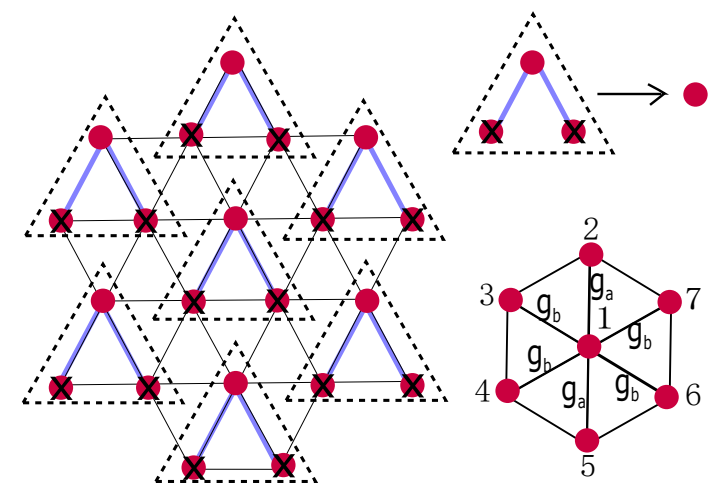

(a)

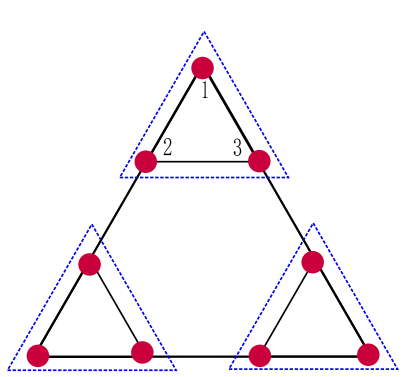

(b)

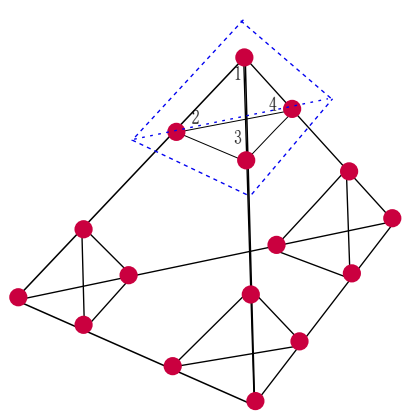

(c)
FIG. 1. Schematic illustration of QRG transformation for the (a) triangular lattice and (b)(c) Sierpiński fractal lattices.

Typically, it is not easy to obtain the analytical solutions of these high-dimensional systems. Even if the numerical method such as Monte-Carlo simulation is applicable [30], the calculation is computationally expensive. The QRG method is a analytical treatment for studying the QPTs, especially has advantages in estimating the quantum critical points and scaling behaviors. The main idea of QRG method is to eliminate or thin the degrees of freedom of the many-body systems through a recursive procedure until a tractable situation is reached. According to the Kadanoff's block method [8, 10, 11], a spin chain can be split into blocks, which means that the Hamiltonian is decomposed into the block Hamiltonian and interacting (interblock) Hamiltonian. The low-lying eigenstates of each block Hamiltonian are applied to construct the basis for renormalized Hilbert space. In this way, the full Hamiltonian is projected onto the renormalized space to achieve an effective Hamiltonian with structural similarity to the original one. As long as the 
thermodynamic limit is touched by increasing the RG iterations, the global properties of the system can be captured.

In the novel QRG method, for the purpose of preserving the symmetry of system and the structural similarity of Hamiltonian, not all the terms inside a block is included in the QRG transformation [17], which is used to significantly improve the estimation precision about the critical point. The procedure of QRG transformation for the triangular lattice is shown in Fig. 1(a). The entire system is covered by blocks of three sites which are renormalized to be a single one. After performing the renormalization, two types of coupling strengths $g_{a}$ and $g_{b}$ replace the original one $g$, which means that the coupling strength becomes highly anisotropic. In Ref. [17], the authors have proposed that the renormalized coupling strength for triangular lattice should be a geometric mean of all coupling strengths and choose the hexagon consisting of seven sites as a basic cluster. Then the renormalized coupling strength can be obtained by

$$
g_{\mathrm{t}}^{\prime}=g_{a}^{2 / 6} g_{b}^{4 / 6}=2^{1 / 3} g^{2}\left(1+g^{2}\right)^{1 / 6}\left(g+\sqrt{1+g^{2}}\right)^{2 / 3} .
$$

In this way, the triangular lattice with $7 \times\left(\lambda^{n}\right)^{d}$ sites can be effectively represented by a seven-site cluster after completing the $n$th RG iteration step, where $d=2$ is the dimension of triangular lattice and $\lambda=\sqrt{3}$ is the scale of the length of the side for each RG iteration. The critical point $g_{\mathrm{c}}^{t}$ corresponding to the nontrivial fixed point is obtained by solving $g^{\prime}=g$, i.e., $g_{\mathrm{c}}^{\mathrm{t}} \approx 0.539$. Similarly, we also study the transverse-field quantum Ising model on the Sierpiński fractal lattices with Hausdorff dimension $d_{\mathrm{H}}=\log (\kappa+1) / \log 2$ where $\kappa=2$ or 3 is the spatial dimension. The procedures of QRG transformation for the Sierpiński triangular lattice $\left(d_{\mathrm{H}}=1.585\right)$ and Sierpiński pyramid lattice $\left(d_{\mathrm{H}}=2\right)$ are depicted in Fig. 1(b) and Fig. 1(c), respectively [17]. It can be observed that the basic cluster in the Sierpiński triangular lattice is a triangle containing three sites, and for the Sierpiński pyramid lattice, it is a pyramid containing four sites. It is not difficult to find that after $n$th RG iterations the Sierpiński triangular (or pyramid) lattice with $3 \times \lambda_{\mathrm{f}}^{1.585 n}$ (or $4 \times \lambda_{\mathrm{f}}^{2 n}$ ) sites is represented by a three (or four)-site cluster, where $\lambda_{\mathrm{f}}=2$. Therefore, the renormalized coupling strengths for the fractal lattices can be obtained as follows

$$
g_{\mathrm{f}}^{\prime}=g^{(3 \kappa+1) /(\kappa+1)}\left(1+g^{2}\right)^{\kappa(\kappa-1) / 2(\kappa+1)} .
$$

The critical points of the Sierpiński triangular lattice and Sierpiński pyramid lattice are given as $g_{\mathrm{c}}^{\mathrm{St}} \approx 0.869$ and $g_{\mathrm{c}}^{\mathrm{Sp}} \approx 0.786$, respectively. Moreover, the correlation length exponents $\nu$ for the triangular lattice, Sierpiński triangular lattice and Sierpiński pyramid lattice can be calculated as follows

$$
\begin{aligned}
& \nu_{\mathrm{t}}^{-1}=\left.\log _{\sqrt{3}} \frac{\mathrm{d} g_{\mathrm{t}}^{\prime}}{\mathrm{d} g}\right|_{g_{\mathrm{c}}^{\mathrm{t}}}, \\
& \nu_{\mathrm{f}}^{-1}=\left.\log _{2} \frac{\mathrm{d} g_{\mathrm{f}}^{\prime}}{\mathrm{d} g}\right|_{g_{\mathrm{c}}} .
\end{aligned}
$$

The results are $\nu_{\mathrm{t}} \simeq 0.630, \nu_{\mathrm{St}} \simeq 0.720$ and $\nu_{\mathrm{Sp}} \simeq 0.617$, respectively.
Next, we briefly outline the definition of the monogamy of entanglement and the measure of multipartite entanglement in the present study. For an $N$-qubit system with state space $\mathcal{H}_{A_{1}} \otimes \mathcal{H}_{A_{2}} \otimes \cdots \otimes \mathcal{H}_{A_{N}}$, taking the subsystem $A_{1}$ as a "node" [34], if the entanglement between the particles $A_{1}$ and $A_{2}, \cdots, A_{N}$ satisfies the inequality

$$
E_{A_{1} \mid A_{2}, \cdots, A_{N}}^{2} \geq E_{A_{1} A_{2}}^{2}+E_{A_{1} A_{3}}^{2}+\cdots+E_{A_{1} A_{N}}^{2},
$$

with $E_{A_{1} \mid A_{2}, \cdots, A_{N}}$ quantifying the entanglement in the partition $A_{1} \mid A_{2}, \cdots, A_{N}$ and $E_{A_{1} A_{j}}$ quantifying the one in the two-qubit system $A_{1} A_{j}$, then the entanglement measure $E$ obeys the monogamous relation [26]. This monogamy property imposes physical restrictions on unconditional sharability of quantum entanglement between the different parts of a many-body system. According to the Schmit decomposition [31], the subsystem $A_{2}, \cdots, A_{N}$ is equal to a logic qubit $A_{2, \cdots, N}$ for an $N$ qubit pure state $|\psi\rangle_{A_{1} A_{2}, \cdots, A_{N}}$. As an example, the entanglement of formation $E_{\mathrm{f}}\left(A_{1} \mid A_{2}, \cdots, A_{N}\right)$ can be derived by using the analytical formula for a two-qubit state $\rho_{A B}[32]$

$$
E_{\mathrm{f}}\left(\rho_{A B}\right)=h\left(\frac{1+\sqrt{1-C_{A B}^{2}}}{2}\right),
$$

where $h(x)=-x \log _{2} x-(1-x) \log _{2}(1-x)$ is the binary entropy and $C_{A B}=\max \left\{0, \sqrt{\lambda_{1}}-\sqrt{\lambda_{2}}-\sqrt{\lambda_{3}}-\sqrt{\lambda_{4}}\right\}$ is the concurrence [33] with decreasing nonnegative $\lambda_{i} \mathrm{~S}$ being the eigenvalues of the matrix $\rho_{A B}\left(\sigma_{y} \otimes \sigma_{y}\right) \rho_{A B}^{*}\left(\sigma_{y} \otimes\right.$ $\left.\sigma_{y}\right)$. The squared entanglement of formation has been found to obey the monogamy inequality in an arbitrary $N$-qubit mixed state, and a relevant indicator has been proposed to detect the multiqubit entangled states [27], which reads

$$
\tau_{A_{1} \mid A_{2}, \cdots, A_{N}}=E_{\mathrm{f}}^{2}\left(\rho_{A_{1} \mid A_{2}, \cdots, A_{N}}\right)-\sum_{j \neq 1}^{N} E_{\mathrm{f}}^{2}\left(\rho_{A_{1} A_{j}}\right) .
$$

By utilizing this measure, we can not only explore the QPTs of many-body systems, but also examine the performance of multipartite entanglement in different phases.

\section{II.1. triangular lattice}

Now, we investigate the multipartite entanglement for the transverse-field quantum Ising model on the triangular lattice by employing the QRG method. Since the basic cluster contains seven sites, as shown in Fig. 1(a), we choose the central site (labeled by 1) as the "node" [34], and calculate the seven-partite entanglement $\tau_{1 \mid 2, \cdots, 7}$ for studying the performances of multipartite entanglement in the QPT. The Hamiltonian of basic cluster can be written as

$$
H=-g\left(\sum_{i=2}^{7} \sigma_{1}^{z} \sigma_{i}^{z}+\sum_{i=2}^{6} \sigma_{i}^{z} \sigma_{i+1}^{z}+\sigma_{2}^{z} \sigma_{7}^{z}\right)-\sum_{i=1}^{7} \sigma_{i}^{x} .
$$


The density matrix is given by $\rho=\left|\psi_{0}\right\rangle\left\langle\psi_{0}\right|$, where $\left|\psi_{0}\right\rangle$ is the ground state of the Hamiltonian of the basic cluster. Then we can calculate the seven-partite entanglement according to Eq. 7, namely,

$$
\tau_{1 \mid 2, \cdots, 7}=E_{\mathrm{f}}^{2}\left(\rho_{1 \mid 2, \cdots, 7}\right)-\sum_{j \neq 1}^{7} E_{\mathrm{f}}^{2}\left(\rho_{1 j}\right) .
$$

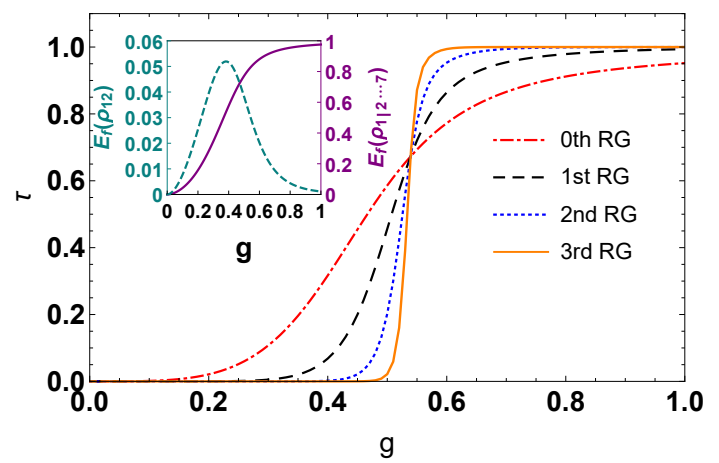

FIG. 2. The evolution of multipartite entanglement $\tau$ versus coupling strength $g$ for different RG iterations on the triangular lattice. The inset depicts the bipartite entanglement $E_{\mathrm{f}}\left(\rho_{12}\right)$ (green dashed line) and $E_{\mathrm{f}}\left(\rho_{1 \mid 2 \cdots 7}\right)$ (purple solid line) as functions of coupling strength $g$ for the zeroth RG iteration.

Based on Eqs. 2 and 9, we compute the seven-partite entanglement between seven sites in the basic cluster which can represent different system sizes after completing the corresponding RG iterations. The seven-partite entanglement $\tau$ as a function of $g$ for different RG iterations on the triangular lattice is plotted in Fig. 2. It can be observed that these curves cross each other at the critical point and two different saturated values of multipartite entanglement associated with two phases: the ferromagnetic phase $\left(g>g_{\mathrm{c}}^{\mathrm{t}}\right)$ and the paramagnetic phase $\left(g<g_{\mathrm{c}}^{\mathrm{t}}\right)$ are developed. As the size of system becomes large, the two phases are diverged more clearly. In particular, the saturated value of multipartite entanglement in the ferromagnetic phase approaches the maximum. Here, in order to provide a possible physical explanation, we display the bipartite entanglements of formation $E_{\mathrm{f}}\left(\rho_{12}\right)$ and $E_{\mathrm{f}}\left(\rho_{1 \mid 2 \cdots 7}\right)$ as functions of coupling strength $g$ for the zeroth $\mathrm{RG}$ iteration in the inset of Fig. 2. The bipartite entanglement $E_{\mathrm{f}}\left(\rho_{12}\right)$ first increases from zero to the maximum and then decreases to zero monotonically, while another one $E_{\mathrm{f}}\left(\rho_{1 \mid 2 \cdots 7}\right)$ increases monotonically with $g$ until the saturated value is arrived. As the coupling strength grows from zero, the increased probability for the spin pair staying at the entangled state leads to the generation of bipartite entanglement. Only when the competition between the interaction and quantum fluctuation reaches a counterbalance at the critical point, the bipartite entanglement $E_{\mathrm{f}}\left(\rho_{12}\right)$ reaches its maximum [35], agreeing with the results of Ref. [13]. Then the exchange couplings play a dominant role and keep the system staying at the ferromagnetic phase, which results in the decrease of bipartite entanglement between two
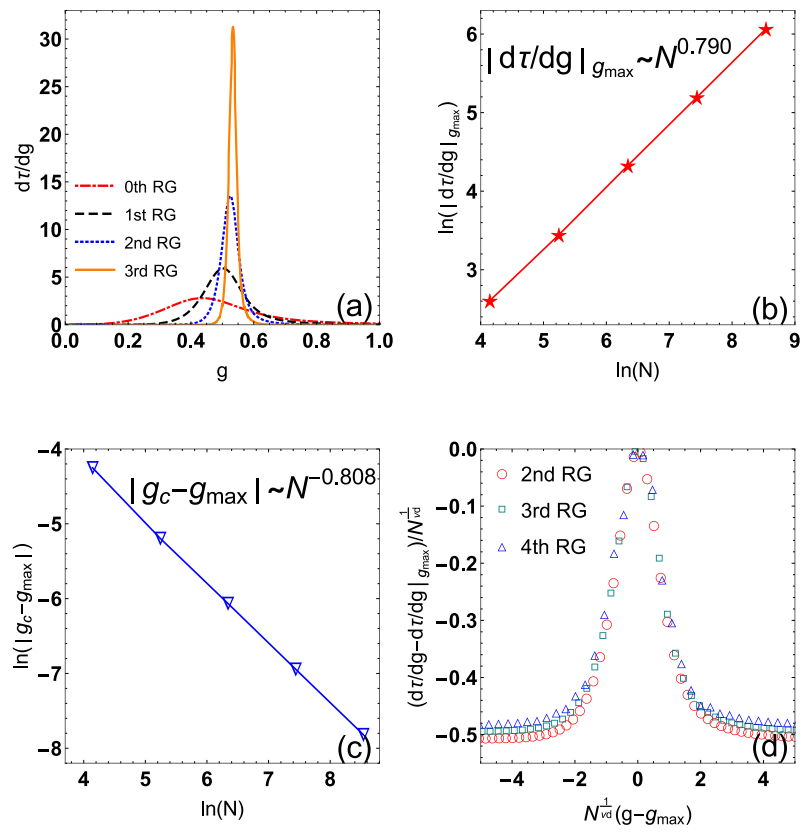

FIG. 3. (a) First derivative of multipartite entanglement $\mathrm{d} \tau / \mathrm{d} g$ versus $g$ for different $\mathrm{RG}$ iterations on the triangular lattice. (b) The logarithm of the absolute value of maximum $\ln |\mathrm{d} \tau / \mathrm{d} g|$ versus the logarithm of the triangular lattice size $\ln (N)$, which is linear and shows a scaling behavior. (c) The scaling behavior of $g_{\max }$ in terms of system size $N$ for the triangular lattice, where $g_{\max }$ is the position of the maximum derivative of multipartite entanglement. (d) The finite-size scaling through renormalization treatment with the correlation length exponent $\nu=0.63$ for the multipartite entanglement. The curves corresponding to different system sizes approximately collapse onto a single one for this triangular lattice.

neighboring sites. We can find the reason why the multipartite entanglement approaches the maximum in the ferromagnetic phase according to Eq. 7. On the one hand, the increase of coupling strength brings the value of quantum entanglement $E_{\mathrm{f}}\left(\rho_{1 \mid 2 \cdots 7}\right)$ up to the maximum. On the other hand, the bipartite entanglements between two neighboring sites are very little since the strong coupling strength. It can be concluded that the multipartite entanglement is not maximal at the critical point as the bipartite entanglement be, and the multipartite entanglement is richer in the ferromagnetic phase than the paramagnetic phase.

Although above interpretation is superficial, it provides inspiration for further understanding. At zero field the model exhibits ferromagnetic behavior with net magnetization in the $z$ direction. The ground state is twofold degenerate and a product state with spins pointing in the $z$ direction, i.e. $|+\rangle=|\uparrow \uparrow \cdots\rangle$ or $|-\rangle=|\downarrow \downarrow \cdots\rangle$. In the large-field limit, ground state is also a product state with all spins being polarized to the direction of field. Although no bipartite entanglement exists in both cases, there is another possible solution for the ground state at zero field, namely, the superposition of the degenerate states, which may be a Greenberger-Horne-Zeilinger 
(GHZ)-like state $|G\rangle=1 / \sqrt{2}(|+\rangle+|-\rangle)$ with genuine multipartite entanglement [36]. Therefore, the multipartite entanglement approaches the maximum in the ferromagnetic phase, and these results may provide us a further insight in the entanglement distribution and QPT for many-body systems.

More information on the location and the order of the QPT can be obtained by consideration of the derivatives of the multipartite entanglement with respect to the coupling strength. We plot the derivatives of multipartie entanglement $\mathrm{d} \tau / \mathrm{d} g$ as a function of $g$ for different RG iterations in Fig. 3(a). It can be seen from Fig. 3(a) that the first derivative of the multipartie entanglement exhibits a nonanalytic behavior, which indicates that the QPT of this system is a second-order QPT. The scaling behavior of the maximum of $\mathrm{d} \tau / \mathrm{d} g$ versus $N$ is displayed in the Fig. 3(b), which is a linear behavior of $\ln \left(|\mathrm{d} \tau / \mathrm{d} g|_{g_{\max }}\right)$ versus $\ln (N)$. Based on numerical analysis, we can obtain $|\mathrm{d} \tau / \mathrm{d} g|_{g_{\max }} \sim N^{\mu_{1}^{\prime}}$ where the critical exponent $\mu_{1}^{\prime} \simeq 0.790$. It has been found that the correlation length exponent is the inverse of critical exponent in the one-dimensional spin chain systems [37, 38]. For this triangular lattice, the relation between the correlation length exponent and critical exponent has a new form. The correlation length exponent $\nu$ gives the divergent behavior of correlation length in the vicinity of $g_{\mathrm{c}}$, i.e., $\xi \sim\left|g-g_{\mathrm{c}}\right|^{-\nu}$. Under the RG transformations, the correlation length scales as $\xi \rightarrow \xi_{n}=\xi / \lambda^{n}$, where $\lambda=\sqrt{3}$ is the scale of the length of the side for each RG iteration and related to $N$, i.e. $7 \times\left(\lambda^{n}\right)^{d}=N$. For the $n$th RG iteration, the renormalized coupling strength $g_{n}$ is still a function of original one $g$. Since $\xi_{n} \sim\left|g_{n}-g_{\mathrm{c}}\right|^{-\nu}$ and $|\mathrm{d} \tau / \mathrm{d} g|_{g_{\max }}|\sim| \mathrm{d} g_{n} /\left.\mathrm{d} g\right|_{g_{\mathrm{c}}} \mid$, we can derive that

$$
\left|\frac{\mathrm{d} \tau}{\mathrm{d} g}\right|_{g_{\max }} \sim N^{\frac{1}{\nu d}}
$$

Comparing with $|\mathrm{d} \tau / \mathrm{d} g|_{g_{\max }} \sim N^{\mu_{1}}$, the relation of critical exponent and correlation length exponent is obtained, namely $\mu_{1}=1 /(\nu d)$. Furthermore, the value of coupling strength $g_{\max }$ corresponding to the maximum of $\mathrm{d} \tau / \mathrm{d} g$ for each RG iteration gradually tends toward the critical point $g_{\mathrm{c}}$, which indicates another scaling behavior displayed in Fig. 3(c), i.e., $\left|g_{\mathrm{c}}-g_{\max }\right| \sim N^{-\mu_{2}^{\prime}}$ where the critical exponent $\mu_{2}^{\prime} \simeq 0.808$. This critical exponent is also related to the correlation length exponent $\nu$ in the vicinity of the critical point. The scaling of the position of maximum $g_{\max }$ comes from the behavior of the correlation length $\xi$ near the critical point. As the thermodynamic limit is approached, the correlation length $\xi \sim N^{1 / d}$. Comparing with $\xi \sim\left|g-g_{c}\right|^{-\nu}$, the scaling form $\left|g_{\mathrm{c}}-g_{\max }\right| \sim N^{-1 /(\nu d)}$ can be obtained, which implies that $\mu_{2}=1 /(\nu d)$. We can observe that the second critical exponent is in good agreement with the first one $\mu_{1}=\mu_{2}=1 /\left(\nu_{\mathrm{t}} d\right) \simeq 0.794$ where $\nu_{\mathrm{t}}$ is the correlation length exponent for the triangular lattice as shown in Eq. 4. It is noted that the above relation can also be proved by the numerical results $\mu_{1}^{\prime} \simeq 0.790$ and $\mu_{2}^{\prime} \simeq 0.808$. Based on the divergence of derivative of multipartite entanglement, we plot $\left(\mathrm{d} \tau / \mathrm{d} g-\mathrm{d} \tau /\left.\mathrm{d} g\right|_{g_{\max }}\right) / N^{\frac{1}{\nu d}}$ versus
$N^{\frac{1}{\nu d}}\left(g-g_{\max }\right)$ for different RG iterations in Fig. 3(d). These curves for different system sizes approximately collapse onto a single one, which is a manifestation of the existence of finite-size scaling for the multipartite entanglement $[4,39]$. We can conclude that the multipartite entanglement is a good indicator to signify the criticality of the transverse-field quantum Ising model on the triangular lattice.

\section{II.2. Sierpiński fractal lattice}
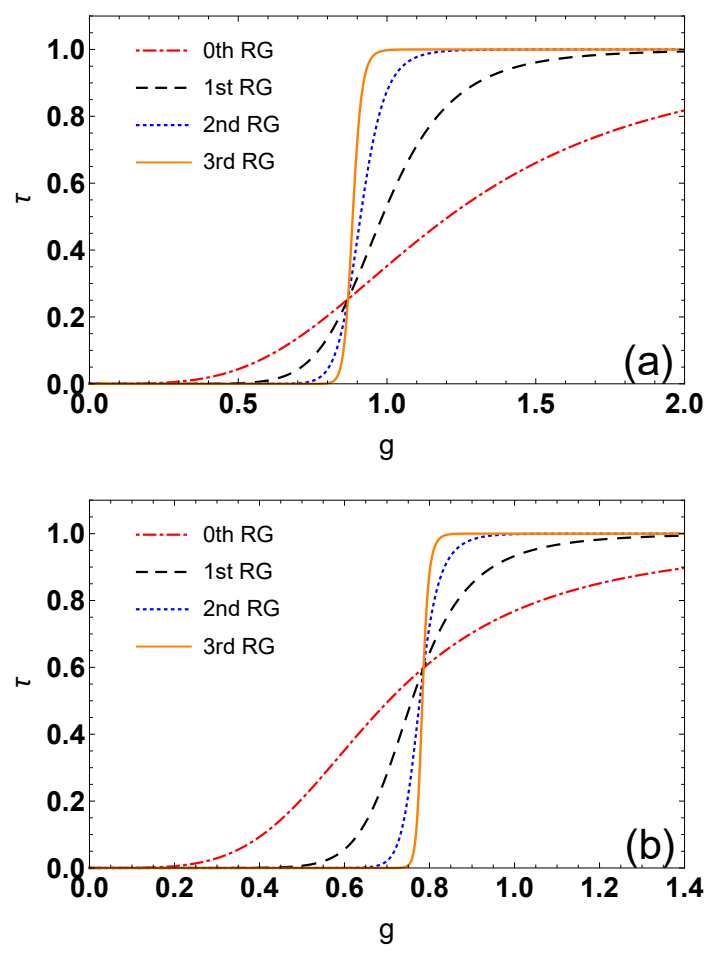

FIG. 4. The evolutions of multipartite entanglement $\tau$ versus $g$ for different RG iterations on the (a) Sierpiński triangular lattice and (b) Sierpiński pyramid lattice.

Next, we consider the transverse-field Ising model on the fractal lattices which are the generalizations of the Sierpiński pyramid in $\kappa$ spatial dimensions. For $\kappa=2$ and $\kappa=3$, the fractal lattices are Sierpiński triangle and pyramid lattices, respectively, as depicted in Fig. 1(b) and Fig. 1(c), whose Hausdorff dimensions can be calculated by $d_{\mathrm{H}}=\log (\kappa+1) / \log 2$. Here, we choose the site labeled by 1 as the "node", and according to the numbers of sites in basic clusters of fractal lattices, we investigate the tripartite entanglement for Sierpiński triangle lattice and four-partite entanglement for Sierpiński pyramid lattice, respectively. The renormalized tripartite and fourpartite entanglements can be obtained from Eqs. 3 and 7 . The results about multipartite entanglement versus the reduced coupling strength $g$ for different RG iterations on the Sierpiński triangular and pyramid lattices are displayed in Fig. 4. It is clearly observed from Fig. 4 that the evolutions of multipartite entanglement on the frac- 

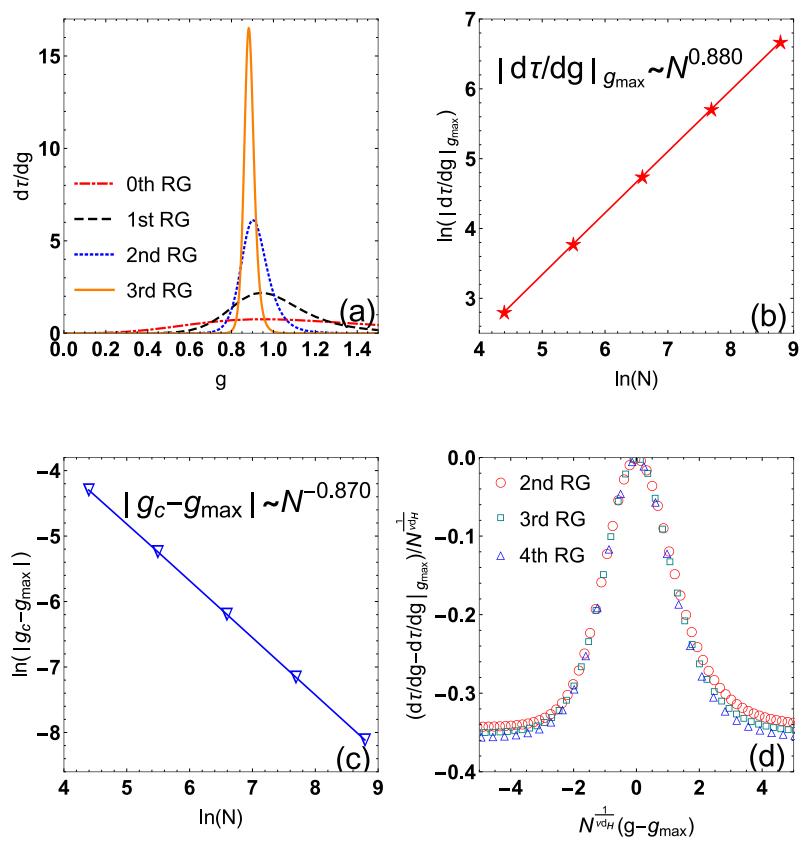

FIG. 5. (a) The first derivative of multipartite entanglement $\mathrm{d} \tau / \mathrm{d} g$ versus $g$ for different $\mathrm{RG}$ iterations on the Sierpiński triangular lattice. (b) The logarithm of the absolute value of maximum $\ln |\mathrm{d} \tau / \mathrm{d} g|$ versus the logarithm of the Sierpiński triangular lattice size $\ln (N)$, which is linear and shows a scaling behavior. (c) The scaling behavior of $g_{\max }$ in terms of system size $N$ for the Sierpiński triangular lattice, where $g_{\max }$ is the position of the maximum derivative of multipartite entanglement. (d) The finite-size scaling through renormalization treatment with the correlation length critical exponent $\nu=0.720$ for the multipartite entanglement. The curves corresponding to different system sizes approximately collapse onto a single one for this Sierpiński triangular lattice.

tal lattices are similar to that on the triangular lattice. As the size of the Sierpiński triangular lattice increases, the tripartite entanglement produces two different saturated values that corresponding to two different phases. The paramagnetic order at $g<g_{\mathrm{c}}^{\mathrm{St}}$ induces the quantum fluctuation and leads to the destruction of tripartite entanglement. In contrast, as $g$ becomes large, the ferromagnetic order gradually builds the tripartite entanglement. The performance of four-partite entanglement in the Sierpiński pyramid lattice is analogous to the tripartite entanglement in the Sierpiński triangle lattice, one obvious difference is the positions of intersection points since the critical points of these two fractal lattices are diverse. These two figures reveal that as the thermodynamic limit is touched by increasing the RG iterations, the multipartite entanglement can be used to detect the critical points of the fractal lattices.

The appearance of nonanalytic behavior in some quantity, often accompanied by a scaling behavior, is a feature of second-order QPT. The nonanalytic phenomenons of the first derivative of multipartite entanglement near the critical point and the scaling behaviors for the twodimensional triangular lattice have been shown in Fig. 3. In the following, we pay our attention to the cases of
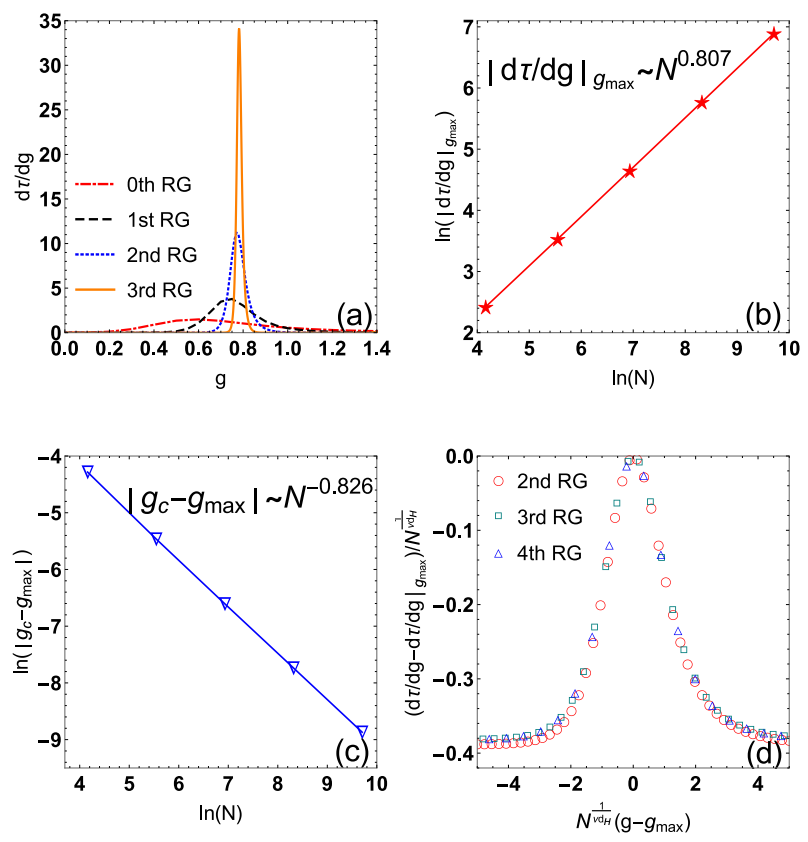

FIG. 6. (a) The first derivative of multipartite entanglement $\mathrm{d} \tau / \mathrm{d} g$ versus $g$ for different RG iteration on the Sierpiński pyramid lattice. (b) The logarithm of the absolute value of maximum $\ln |\mathrm{d} \tau / \mathrm{d} g|$ versus the logarithm of the Sierpiński pyramid lattice size $\ln (N)$, which is linear and shows a scaling behavior. (c) The scaling behavior of $g_{\max }$ in terms of system size $N$ for the Sierpiński pyramid lattice, where $g_{\max }$ is the position of the maximum derivative of multipartite entanglement. (d) The finite-size scaling through renormalization treatment with the correlation length critical exponent $\nu=0.617$ for the multipartite entanglement. The curves corresponding to different system sizes approximately collapse onto a single one for this Sierpiński pyramid lattice.

fractal lattices. The first derivatives of multipartite entanglement $\mathrm{d} \tau / \mathrm{d} g$ versus $g$ for different RG iterations on the Sierpiński triangular and pyramid lattices have been displayed in Fig. 5(a) and Fig. 6(a), respectively. The nonanalytic behaviors of multipartite entanglement near the critical points become more prominent when the sizes of systems increase, which means that the first derivatives of multipartite entanglement are singular near the critical points, and the systems both undergo the second-order QPTs. To further understand the relation between the renormlized multipartite entanglement and QPTs, we explore the finite-size scaling behaviors of multipartite entanglement close to the critical points. The linear behaviors of $\ln \left(|\mathrm{d} \tau / \mathrm{d} g|_{g_{\max }}\right)$ versus $\ln (N)$ are revealed in Figs. 5 (b) and 6(b). Numerical analysis confirmed that the maximum of $\mathrm{d} \tau / \mathrm{d} g$ obeys the following finite-size scaling behavior: $|\mathrm{d} \tau / \mathrm{d} g|_{g_{\max }} \mid \sim N^{\mu^{\prime}}$, where the critical exponent for Sierpiński triangular lattice is $\mu_{3}^{\prime} \simeq 0.880$ as shown in Fig. 5(b) and the one for Sierpiński pyramid lattice is $\mu_{5}^{\prime} \simeq 0.807$ as shown in Fig. 6 (b). The correlation length exhibits exponential behavior near the critical point $g_{\mathrm{c}}$, i.e., $\xi \sim\left|g-g_{\mathrm{c}}\right|^{-\nu}$. After the $n$th iteration, the correlation length scales as $\xi_{n}=\xi / \lambda^{n} \sim\left|g_{n}-g_{\mathrm{c}}\right|^{-\nu}$ with $\lambda_{\mathrm{f}}=2 . N$ and $\lambda_{\mathrm{f}}$ in the fractal lattice have the relation 
$N=N_{0} \lambda_{\mathrm{f}}^{n d_{\mathrm{H}}}$, where $N_{0}=3$ for $\kappa=2$ and $N_{0}=4$ for $\kappa=3$. Since $|\mathrm{d} \tau / \mathrm{d} g|_{g_{\max }}|\sim| \mathrm{d} g_{n} /\left.\mathrm{d} g\right|_{g_{\mathrm{c}}} \mid$, we can derive that

$$
\left|\frac{\mathrm{d} \tau}{\mathrm{d} g}\right|_{g_{\max }} \sim N^{\frac{1}{\nu d_{\mathrm{H}}}} .
$$

Comparing with $|\mathrm{d} \tau / \mathrm{d} g|_{g_{\max }} \sim N^{\mu}$, the relation of critical exponent and correlation length exponent can be obtained $\mu=1 /\left(\nu d_{\mathrm{H}}\right)$. It means that $\mu_{3}=$ $1 /\left(\nu_{\mathrm{St}} d_{\mathrm{H}}^{\mathrm{St}}\right) \simeq 0.876$ for Sierpiński triangular lattice and $\mu_{5}=1 /\left(\nu_{\mathrm{Sp}} d_{\mathrm{H}}^{\mathrm{Sp}}\right) \simeq 0.810$ for Sierpiński pyramid lattice. The numerical results listed above $\mu_{3}^{\prime} \simeq 0.880$ and $\mu_{5}^{\prime} \simeq 0.807$ are in good agreement with the analytical results, i.e. $\mu_{3}^{\prime} \simeq \mu_{3}$ and $\mu_{5}^{\prime} \simeq \mu_{5}$.

Furthermore, the value of $g_{\max }$ corresponding to the maximum of $\mathrm{d} \tau / \mathrm{d} g$ for each RG iteration gradually tends toward the critical point $g_{\mathrm{c}}$. It indicates $\left|g_{\mathrm{c}}-g_{\max }\right| \sim$ $N^{-\mu}$, where the critical exponent for Sierpiński triangular lattice is $\mu_{4}^{\prime} \simeq 0.870$ as shown in Fig. 5(c) and the one for Sierpiński pyramid lattice is $\mu_{6}^{\prime} \simeq 0.826$ as shown in Fig. 6(c). These critical exponents $\mu_{4}$ and $\mu_{6}$ are directly related the correlation length exponents in the vicinities of the critical points. The correlation length is related to the size of the system in the thermodynamic limit, i.e., $\xi \sim N^{1 / d_{\mathrm{H}}}$. Since $\xi \sim\left|g-g_{c}\right|^{-\nu}$, then the scaling form $\left|g_{\mathrm{c}}-g_{\max }\right| \sim N^{-1 /\left(\nu d_{\mathrm{H}}\right)}$ can be obtained, which implies that $\mu=1 /\left(\nu d_{\mathrm{H}}\right)$. That is to say, for the Sierpiński triangular lattice, $\mu_{4}=1 /\left(\nu_{\mathrm{St}} d_{\mathrm{H}}^{\mathrm{St}}\right) \simeq 0.876$ and for the Sierpiński pyramid lattice, $\mu_{6}=1 /\left(\nu_{\mathrm{Sp}} d_{\mathrm{H}}^{\mathrm{Sp}}\right) \simeq 0.810$. The numerical results are also consistent with the analytical results, i.e., $\mu_{4}^{\prime} \simeq \mu_{4}$ and $\mu_{6}^{\prime} \simeq \mu_{6}$.

Finally, it is possible to make all the data from different RG iterations collapse onto a single curve by choosing a suitable scaling function and taking into account the distance of the maximum of the derivatives of multipartite entanglement from the critical point $[4,39]$. We display $\left(\mathrm{d} \tau / \mathrm{d} g-\mathrm{d} \tau /\left.\mathrm{d} g\right|_{g_{\max }}\right) / N^{\frac{1}{\nu d_{\mathrm{H}}}}$ versus $N^{\frac{1}{\nu d_{\mathrm{H}}}}\left(g-g_{\max }\right)$ for different RG iterations on the Sierpiński triangular lattice in Fig. 5(d) and on the Sierpiński pyramid lattice in Fig. 6(d). These curves approximately collapse onto a single universal one, which is a manifestation of the existence of finite-size scaling for the multipartite entanglement. These results justify that the RG implementation of multipartite entanglement truly capture the critical behaviors of the transverse-field quantum Ising model on the fractal lattices.

It is well known that the cornerstone of the theory of critical phenomena is the universality, which indicates that the critical behavior is depend on the dimension of system and the symmetry of chosen order parameter $[1,4]$. From above discussions, it can be confirmed that the critical behaviors of these lattices depend on their dimensions. Furthermore, we want to point out that the multipartite entanglement may be a better choice than bipartite entanglement or quantum correlations for studying the many-body systems. On the one hand, the bipartite entanglement has limited ability to capture the characters of the many-body systems. A typical example is the $N$-qubit GHZ state $|G\rangle=1 / \sqrt{2}(|\uparrow \uparrow \cdots \uparrow\rangle+$ $|\downarrow \downarrow \cdots \downarrow\rangle)$, which has been proved to be an $N$-partite entangled state. However, its reduced density matrix of two spins $(i$ and $j) \rho_{i j}=1 / 2(|\uparrow \uparrow\rangle\langle\uparrow \uparrow|+| \downarrow \downarrow\rangle\langle\downarrow \downarrow|)$ is a separable mixed state and has no entanglement. In Fig. 2, the bipartite entanglement only reaches the maximum near the critical point, while the multipartite entanglement reaches the maximum in a more extensive region. The multipartite entanglement in ferromagnetic phase may be a valuable resource for the quantum information processing tasks. We may lose this important information and have less chance to know the entanglement distribution of the many-body system if we only consider the bipartite entanglement. On the other hand, although the bipartite entanglement has been successfully proved to capture the quantum critical points of some many-body systems, it has been indicated that the bipartite entanglement may fail to characterize the real quantum critical points [3, 40, 41]. For example, the concurrence may show no special behavior at the real critical point of the one-dimensional frustrated spin-1/2 Heisenberg model [40]. In this sense, the multipartite entanglement provides a global view and more physical insights into the characters of many-body systems and may have some advantages over bipartite entanglement or quantum correlation for studying the many-body systems [36, 42].

\section{QUANTUM COHERENCE AND QUANTUM PHASE TRANSITIONS IN LATTICES}

In this section, we choose the quantum coherence as an indicator to study the QPTs in the transverse-field quantum Ising model on the triangular lattice and fractal lattices by using the QRG method. It is noted that the existence of QPT is independent of the chosen physical quantity. In order to quantify the amount of quantum coherence, the $l_{1}$-norm and quantum relative entropy coherence have been proposed in Ref. [29]. Besides, some other effective quantifiers of quantum coherence, such as the quantum coherence based on the trace distance and quantum Jensen-Shannon divergence (QJSD) have been put forward in the later works [43-45]. Here, we choose the quantum coherence based on the QJSD [45] to study the QPTs of lattices. The QJSD is a measure of distinguishability between two quantum states [46]

$$
J(\rho, \sigma)=S\left(\frac{\rho+\sigma}{2}\right)-\frac{S(\rho)+S(\sigma)}{2},
$$

where $S(\rho)=-\operatorname{Tr} \rho \log _{2} \rho$ is the von Neumann entropy. In Ref. [47], the metric character of QJSD has been discussed and a true metric based on the square root of QJSD has been proposed as follows

$$
D(\rho, \sigma)=\sqrt{J(\rho, \sigma)} .
$$

It is noted that this metric verifies the triangle inequality in addition to satisfying the distance axioms, and it is a valuable tool since its metric properties. Moreover, it has been proven to be true for qubit and qudit systems 
$[45,48,49]$, and the measure of quantum coherence based on the square root of the QJSD is given by

$$
C(\rho)=\sqrt{S\left(\frac{\rho+\rho_{\mathrm{dia}}}{2}\right)-\frac{S(\rho)+S\left(\rho_{\mathrm{dia}}\right)}{2}}
$$

where $\rho_{\text {dia }}$ is the incoherent state obtained from $\rho$ by deleting all off-diagonal elements [29]. Quantum coherence are usually ascribed to the off-diagonal elements of a density matrix with respect to a reference basis. We fix the computational basis $\{|0\rangle,|1\rangle\}$ as the reference basis, where $|0\rangle$ and $|1\rangle$ are the eigenvectors of spin operator $\sigma^{z}$.

\section{III.1. triangular lattice}

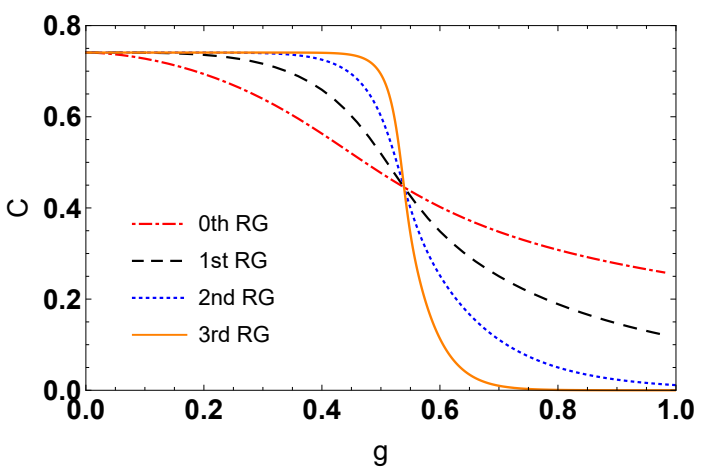

FIG. 7. The evolution of quantum coherence $C$ versus $g$ for different $\mathrm{RG}$ iterations on the triangular lattice.

First, we investigate the quantum coherence between two nearest-neighbor sites on the triangular lattice. As shown in Fig. 1(a), the basic cluster contains seven sites, for simplicity, we apply the quantum coherence $C\left(\rho_{12}\right)$ between sites 1 and 2 to study the QPT, where the reduced density matrix $\rho_{12}$ can be obtained by tracing over the sites $3, \cdots, 7$. The quantum coherence $C$ as a function of $g$ for different RG iterations on the triangular lattice is plotted in Fig. 7. By comparing Fig. 2, it is clear that two different saturated values of quantum coherence are developed, however, the behaviors of quantum coherence for two different phases are completely opposite to the ones of multipartite entanglement. This is due to the fact that the quantum coherence is basis-dependent. We choose the eigenvectors of $\sigma^{z}$ as the reference basis and call it $\sigma^{z}$-basis. When the coupling strength $g$ is small enough, the external field induces the quantum fluctuation and lead to all the spins being polarized along the direction of the field, i.e. the $x$ axis. It means that the $\sigma^{x}$ terms contribute to the off-diagonal elements of density matrix, which leads the generation of quantum coherence in the $\sigma^{z}$-basis [50]. Then a saturated value of quantum coherence is reached in the thermodynamic limit. As $g$ increases, the exchange coupling gradually plays a dominant role and keeps the system staying at the ferromagnetic phase, the contribution from the $\sigma^{x}$ terms for quantum coherence almost disappears. Therefore, the quantum coherence tends to zero in ferromagnetic phase after enough RG iterations.
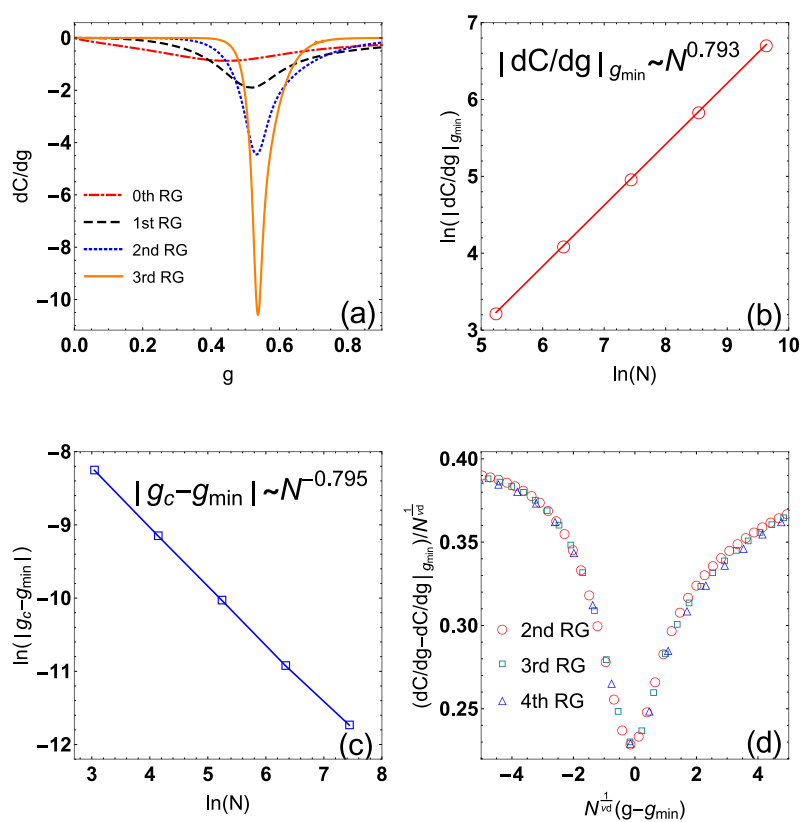

FIG. 8. (a) First derivative of quantum coherence $\mathrm{d} C / \mathrm{d} g$ versus $g$ for different $R G$ iteration on the triangular lattice. (b)The logarithm of the absolute value of minimum $\ln |\mathrm{d} C / \mathrm{d} g|$ versus the logarithm of the triangular lattice size $\ln (N)$, which is linear and shows a scaling behavior. (c) The scaling behavior of $g_{\mathrm{min}}$ in terms of system size $N$ for the triangular lattice, where $g_{\min }$ is the position of the minimum derivative of quantum coherence. (d) The $\left(\mathrm{d} C / \mathrm{d} g-\mathrm{d} C /\left.\mathrm{d} g\right|_{g_{\min }}\right) / N^{1 /(\nu d)}$ versus $N^{1 /(\nu d)}\left(g-g_{\min }\right)$ for different RG iterations on triangular lattice where the correlation length critical exponent $\nu=0.630$. The curves corresponding to different system sizes approximately collapse onto a single one.

In order to obtain the precise location of critical point and the order of QPT, we look at the derivatives of the quantum coherence $\mathrm{d} C / \mathrm{d} g$ as a function of $g$ for different RG iterations in Fig. 8(a). It is quite clear from Fig. 8(a) that the first derivative of the quantum coherence exhibits a nonanalytic behavior in the vicinity of the critical point, which is a feature of the secondorder QPT. The position of the minimum of $\mathrm{d} C / \mathrm{d} g$ is gradually close to the critical point as the size of system increases. A linear behavior of $\ln \left(|\mathrm{d} C / \mathrm{d} g|_{g_{\text {min }}}\right)$ versus $\ln (N)$ is displayed in Fig. 8(b). The critical exponent $\mu_{1}^{\prime \prime}$ for this scaling behavior is $|\mathrm{d} C / \mathrm{d} g|_{g_{\min }} \sim N^{\mu_{1}^{\prime \prime}}$ where $\mu_{1}^{\prime \prime} \simeq 0.793$. Another scaling behavior is shown in Fig. $8\left(\right.$ c), i.e., $\left|g_{\mathrm{c}}-g_{\min }\right| \sim N^{-\mu_{2}^{\prime \prime}}$ where the critical exponent $\mu_{2}^{\prime \prime} \simeq 0.795$. Using the similar analysis in the previous section, we can obtain the relation between the correlation length exponent and critical exponent for the triangular lattice, namely, $\mu_{1}=\mu_{2}=1 /\left(d \nu_{\mathrm{t}}\right) \simeq 0.794$, which indicates the numerical result is consistent with the analytical one, i.e. $\mu_{1}^{\prime \prime} \simeq \mu_{2}^{\prime \prime} \simeq \mu_{1}$. In Fig. $8(\mathrm{~d})$, we plot $\left(\mathrm{d} C / \mathrm{d} g-\mathrm{d} C /\left.\mathrm{d} g\right|_{g_{\min }}\right) / N^{\frac{1}{\nu d}}$ versus $N^{\frac{1}{\nu d}}\left(g-g_{\min }\right)$ for different RG iterations. All the data from different $N$ 
collapse onto a single curve, which provides a manifestation of the existence of finite-size scaling for the quantum coherence. It can be concluded that the quantum coherence is a good indicator to signify the criticality of the transverse-field quantum Ising model on the triangular lattice.

The multipartite entanglement and quantum coherence are both able to capture the characteristics of ground state and show special behaviors at the real critical point of the triangular lattice, which lead to the appearance of similar properties. At the same time, these similar properties enable us to obtain consistent critical exponents of multipartite entanglement and quantum coherence, i.e. $\mu_{1}^{\prime} \simeq \mu_{2}^{\prime} \simeq \mu_{1}^{\prime \prime} \simeq \mu_{2}^{\prime \prime}$, which is the presentation of the universality of QPT and also demonstrates that the existence of QPT is independent of the chosen physical quantity.
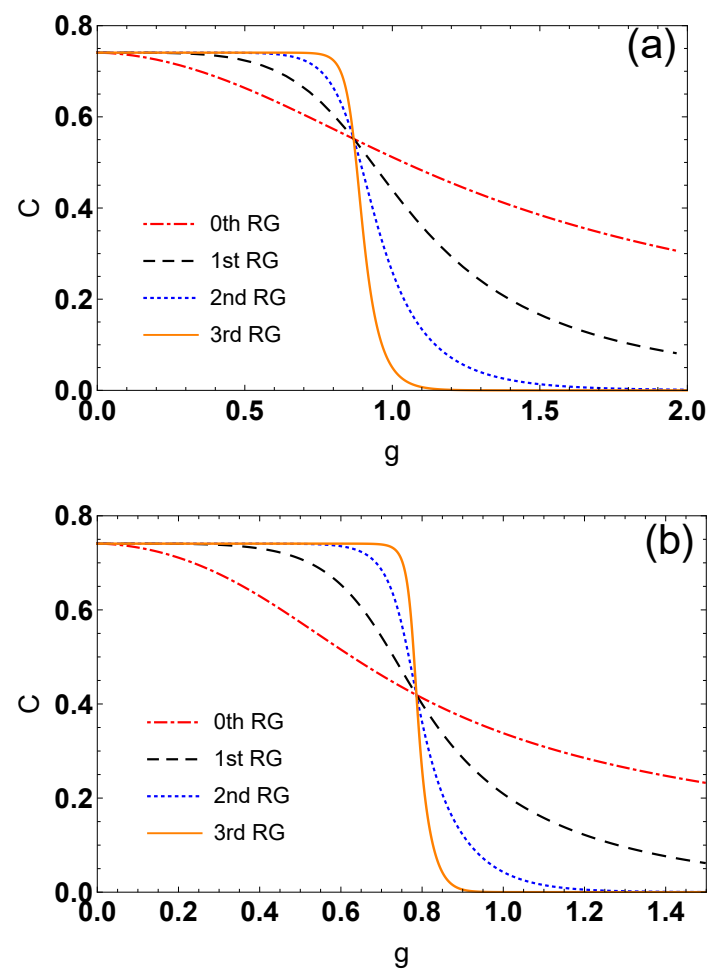

FIG. 9. The evolution of quantum coherence $C$ versus $g$ for different RG iteration on the (a) Sierpiński triangular lattice and (b) Sierpiński pyramid lattice.

\section{III.2. Sierpiński fractal lattice}

Next, we turn to the transverse-field quantum Ising model on the fractal lattices and focus on the quantum coherence between two nearest-neighbor sites which are labeled by 1 and 2 in Figs. 1(b) and 1(c). The results about quantum coherence $C$ versus the reduced coupling strength $g$ for different RG iterations on the Sierpiński triangular and pyramid lattices are shown in Fig. 9. It is found that the evolutions of quantum coherence on
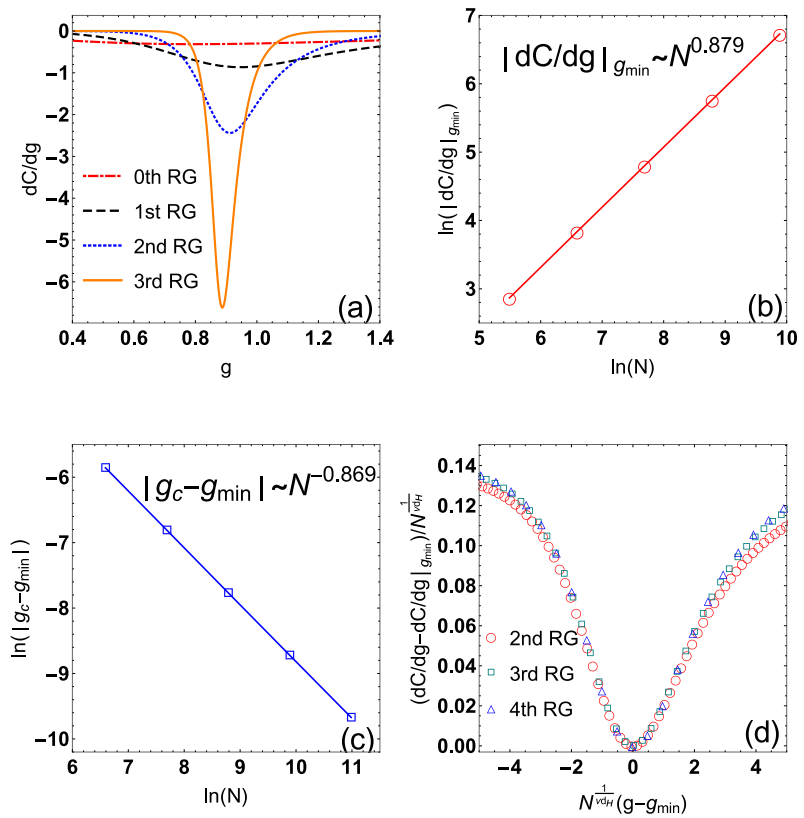

FIG. 10. (a) The first derivative of quantum coherence $\mathrm{d} C / \mathrm{d} g$ versus $g$ for different $R G$ iterations on the Sierpiński triangular lattice. (b) The logarithm of the absolute value of minimum $\ln |\mathrm{d} C / \mathrm{d} g|$ versus the logarithm of the Sierpiński triangular lattice size $\ln (N)$, which is linear and shows a scaling behavior. (c) The scaling behavior of $g_{\min }$ in terms of system size $N$ for the Sierpiński triangular lattice, where $g_{\text {min }}$ is the position of the minimum derivative of quantum coherence. (d) The $\left(\mathrm{d} C / \mathrm{d} g-\mathrm{d} C /\left.\mathrm{d} g\right|_{g_{\min }}\right) / N^{1 /\left(\nu d_{\mathrm{H}}\right)}$ versus $N^{1 /\left(\nu d_{\mathrm{H}}\right)}\left(g-g_{\min }\right)$ for different RG iterations on Sierpiński triangular lattice where the correlation length critical exponent $\nu=0.720$. The curves corresponding to different system sizes approximately collapse onto a single one.

the fractal lattices are similar to that on the triangular lattice. As the size of the lattice increases, the quantum coherence produces two different saturated values that corresponding to two different phases. Quantum coherence is stronger in the paramagnetic phase than the ferromagnetic phase for both of the fractal lattices. The positions of intersection points are different since the critical points of these two fractal lattices are not same. As the thermodynamic limit is touched by increasing the RG iterations, the quantum coherence can be used to detect the critical points of the transverse-field quantum Ising model on fractal lattices.

The nonanalytic features of the first derivatives of quantum coherence at the critical points of the Sierpiński triangular and pyramid lattices are given in Figs. 10(a) and 11(a), respectively. Two systems both exhibit singular properties as the increase of RG iterations. We also explore the finite-size scaling behaviors of renormalized quantum coherence close to the critical points of fractal lattices. The linear behaviors of $\ln \left(|\mathrm{d} C / \mathrm{d} g|_{g_{\min }}\right)$ versus $\ln (N)$ are revealed in Figs. 10(b) and 11(b). The result of numerical analysis confirms that the minimum of $\mathrm{d} C / \mathrm{d} g$ obeys the following finite-size scaling behavior: $|\mathrm{d} C / \mathrm{d} g|_{g_{\min }} \mid \sim N^{\mu^{\prime \prime}}$, where the critical exponent for 

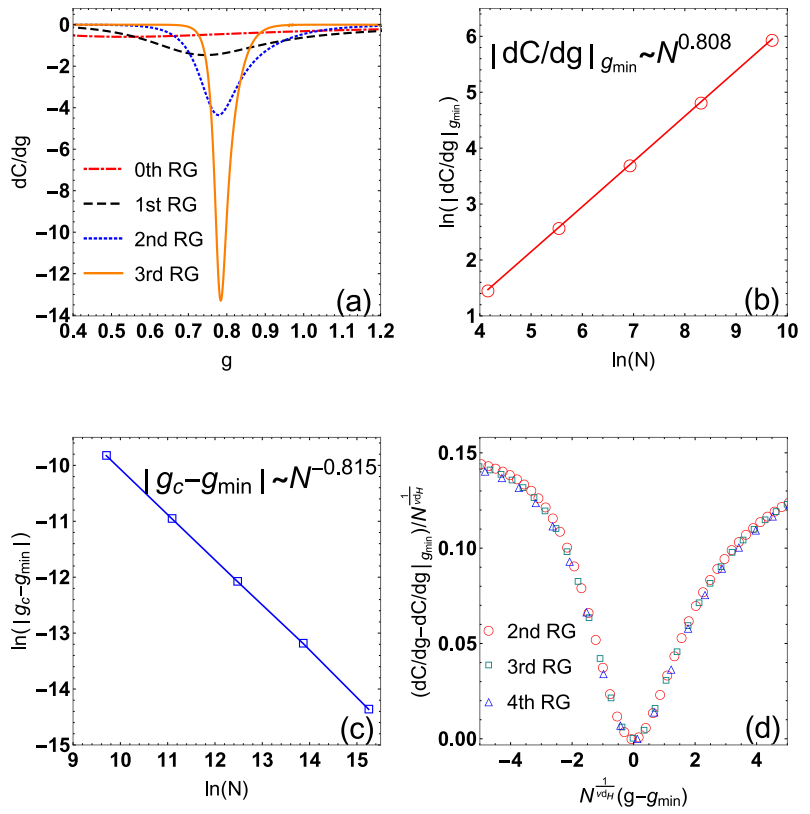

FIG. 11. (a) The first derivative of quantum coherence $\mathrm{d} C / \mathrm{d} g$ versus $g$ for different RG iteration on the Sierpiński pyramid lattice. (b) The logarithm of the absolute value of minimum $\ln |\mathrm{d} C / \mathrm{d} g|$ versus the logarithm of the Sierpiński pyramid lattice size $\ln (N)$, which is linear and shows a scaling behavior. (c) The scaling behavior of $g_{\text {min }}$ in terms of system size $N$ for the Sierpiński pyramid lattice, where $g_{\min }$ is the position of the minimum derivative of quantum coherence. (d) The $\left(\mathrm{d} C / \mathrm{d} g-\mathrm{d} C /\left.\mathrm{d} g\right|_{g_{\min }}\right) / N^{1 /\left(\nu d_{\mathrm{H}}\right)}$ versus $N^{1 /\left(\nu d_{\mathrm{H}}\right)}\left(g-g_{\min }\right)$ for different $R G$ iterations on Sierpiński pyramid lattice where the correlation length critical exponent $\nu=0.617$. The curves corresponding to different system sizes approximately collapse onto a single one.

Sierpiński triangular lattice is $\mu_{3}^{\prime \prime} \simeq 0.879$ as shown in Fig. 10 (b) and the one for Sierpiński pyramid lattice is $\mu_{5}^{\prime \prime} \simeq 0.808$ as shown in Fig. 11(b). Figs 10(c) and $11(\mathrm{c})$ present the results of our analysis for another kind of scaling behavior of $g_{\text {min }}$ in terms of system size $N$, $\left|g_{\mathrm{c}}-g_{\mathrm{min}}\right| \sim N^{-\mu^{\prime \prime}}$, where $\mu_{4}^{\prime \prime} \simeq 0.869$ for Sierpiński triangular lattice and $\mu_{6}^{\prime \prime} \simeq 0.815$ for Sierpiński pyramid lattice. The relations between correlation length exponents and critical exponents of quantum coherence can be analytically obtained as well. In the case of Sierpiński triangular lattice, $\mu_{3}=\mu_{4}=1 /\left(\nu_{\mathrm{St}} d_{\mathrm{H}}^{\mathrm{St}}\right) \simeq 0.876$, and in the case of for Sierpiński pyramid lattice, $\mu_{5}=\mu_{6}=$ $1 /\left(\nu_{\mathrm{Sp}} d_{\mathrm{H}}^{\mathrm{Sp}}\right) \simeq 0.810$. The numerical results are in agreement with the analytical ones. It can be seen from Figs. $10(\mathrm{~d})$ and $11(\mathrm{~d})$ that the curves corresponding to different sizes of system clearly collapse on a single universal curve. These results justify that the RG implementation of quantum coherence truly capture the critical behaviors of the transverse-field quantum Ising model on the fractal lattices.

We want to emphasize that the study of quantum coherence on fractal lattices provides us more insights into the characteristics of the fractal lattices. First, the Hausdorff dimensions determining the relations be- tween correlation length exponents and critical exponents of quantum coherence are confirmed again. Second, the critical exponents of quantum coherence are consistent with the ones of multipartite entanglement, namely $\mu_{3}^{\prime \prime} \simeq \mu_{4}^{\prime \prime} \simeq \mu_{3}^{\prime} \simeq \mu_{4}^{\prime}$ for the Sierpiński triangular lattice and $\mu_{5}^{\prime \prime} \simeq \mu_{6}^{\prime \prime} \simeq \mu_{5}^{\prime} \simeq \mu_{6}^{\prime}$ for the Sierpiński pyramid lattice. These results not only demonstrate that the existence of QPT is independent of the chosen physical quantity, but also are the indication of the universality of QPT.

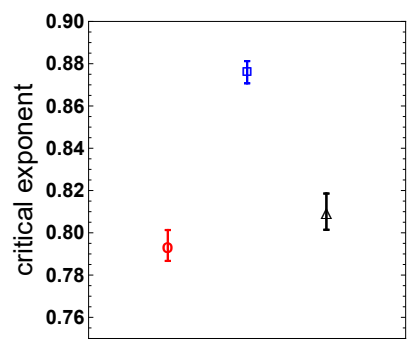

FIG. 12. The critical exponents of the triangular lattice (red circle), Sierpiński triangular lattice (blue square) and Sierpiński pyramid lattice (black triangle) with error bars denoting the simulation errors.

At last, based on the obtained analytical and numerical results of the critical exponents, we display the critical exponents of the triangular lattice and Sierpiński fractral lattices with error bars denoting the simulation errors in Fig. 12. The error bars originate from the standard deviation and indicate the errors between the numerical and analytical results. It can be confirmed again that the numerical values of critical exponents obtained from multipartite entanglement and quantum coherence are consistent with the analytical ones.

\section{CONCLUSIONS}

We investigate the performances of multipartite entanglement and quantum coherence in the quantum phase transitions for transverse-field quantum Ising model on the triangular lattice and Sierpiński fractal lattices by employing the QRG method. It is shown that the quantum criticalities of these high-dimensional models closely relate to the behaviors of the multipartite entanglement and quantum coherence. As the thermodynamic limit is approached, the multipartite entanglement and quantum coherence for these models both develop two different values corresponding to two phases, i.e., the ferromagnetic phase and paramagnetic phase. However, the performances of the multipartite entanglement and quantum coherence in two phases are completely different. The multipartite entanglement in ferromagnetic phase is richer than the one in paramagnetic phase since the GHZ-like state is more likely to exist in the ferromagnetic phase. Nevertheless, the quantum coherence in paramagnetic phase is stronger than the one in ferromagnetic phase, for the reason that the external field may induce 
the quantum fluctuation and lead to some spin being polarized along the direction of the field, and the $\sigma^{x}$ terms contribute to the off-diagonal elements of density matrix, which leads the generation of quantum coherence.

Moreover, the singularities and finite-size scaling behaviors for each lattice can be obtained by calculating the first derivatives of multipartite entanglement and quantum coherence. Although a similar analysis has been performed using the bipartite entanglement in Ref. [13], the authors have only given one kind of scaling behavior for each lattice. In contrast, we have obtained all the scaling behaviors as mentioned in Ref. [4], especially the ones which characterize how the critical points $g_{c}$ of these models are touched as the increase of system sizes. The critical exponents are related to the correlation length exponents and dimensions of lattices, which is due to the fact that the universality of quantum phase transition is dependent on the effective dimension. The multipartite entanglement and quantum coherence are both able to capture the characteristics of ground states and show special behaviors at the critical points, which lead to the appearance of similar properties and consistent critical exponents. It is the presentation of the universality of QPT and also demonstrates that the existence of QPT is independent of the chosen physical quantity.

In general, the multipartite entanglement and quantum coherence are both good indicators to detect the quantum phase transitions in the triangular lattice and Sierpiński fractal lattices. We expect our results to be of interest for a wide range of applications in other highdimensional lattices with help of the QRG method.

\section{ACKNOWLEDGMENTS}

This project was supported by the National Natural Science Foundation of China (Grant No.11274274) and the Fundamental Research Funds for the Central Universities (Grant No.2017FZA3005 and 2016XZZX002-01).
[1] S. Sachdev, Quantum Phase Transitions (Cambridge University Press, England, 1999).

[2] M. A. Nielson and I. L. Chuang, Quantum Computation and Quantum Information (Cambridge University Press,, 2000).

[3] T. J. Osborne and M. A. Nielsen, Phys. Rev. A 66, 032110 (2002).

[4] A. Osterloh, L. Amico, G. Falci, and R. Fazio, Nature 416, 608 (2002).

[5] L. A. Wu, M. S. Sarandy, and D. A. Lidar, Phys. Rev. Lett. 93, 250404 (2004).

[6] L. Amico, R. Fazio, A. Osterloh, and V. Vedral, Rev. Mod. Phys. 80, 517 (2008).

[7] K. G. Wilson, Rev. Mod. Phys 47, 773 (1975).

[8] R. Jafari and A. Langari, Phys. Rev. B 76, 014412 (2007).

[9] F.-W. Ma, S.-X. Liu, and X.-M. Kong, Phys. Rev. A 83, 062309 (2011).

[10] Y. Yao, H.-W. Li, C.-M. Zhang, Z.-Q. Yin, W. Chen, G.-C. Guo, and Z.-F. Han, Phys. Rev. A 86, 042102 (2012).

[11] E. Efrati, Z. Wang, A. Kolan, and L. P. Kadanoff, Rev. Mod. Phys 86, 647 (2014).

[12] M. Usman, A. Ilyas, and K. Khan, Phys. Rev. A 92, 032327 (2015).

[13] Y.-L. Xu, X.-M. Kong, Z.-Q. Liu, and C.-C. Yin, Phys. Rev. A 95, 042327 (2017).

[14] R. Jullien, P. Pfeuty, J. N. Fields, and S. Doniach, Phys. Rev. B 18, 3568 (1978).

[15] K. A. Penson, R. Jullien, and P. Pfeuty, Phys. Rev. B 19, 4653 (1979).

[16] R. Miyazaki, H. Nishimori, and G. Ortiz, Phys. Rev. E 83, 051103 (2011).

[17] A. Kubica and B. Yoshida, arXiv: 1402.0619 (2014).

[18] H. Yi, Phys. Rev. E 88, 014105 (2013).

[19] H. Yi, Phys. Rev. E 91, 012118 (2015).

[20] D. MARKHAM, J. ANDERS, M. HAJDUEK, and V. VEDRAL, Math. Structures Comput. Sci. 23, 441453 (2013).
[21] M. Siomau, AIP Conference Proceedings 1742, 030017 (2016)

[22] D. E. Browne, H. J. Briegel, M. V. D. Nest, R. Raussendorf, and W. Dr, Nat. Phys. 5, 19 (2009).

[23] N. Gisin, G. Ribordy, W. Tittel, and H. Zbinden, Rev. Mod. Phys. 74, 145 (2002).

[24] A. S. Sørensen and K. Mølmer, Phys. Rev. Lett. 86, 4431 (2001).

[25] R. Raussendorf and H. J. Briegel, Phys. Rev. Lett. 86, 5188 (2001).

[26] R. Horodecki, P. Horodecki, M. Horodecki, and K. Horodecki, Rev. Mod. Phys. 81, 865 (2007).

[27] Y. K. Bai, Y. F. Xu, and Z. D. Wang, Phys. Rev. Lett. 113, 100503 (2014).

[28] W. H. Louisell, Quantum Statistical Properties of Radiation (Wiley, 1973).

[29] T. Baumgratz, M. Cramer, and M. B. Plenio, Phys. Rev. Lett. 113, 140401 (2014).

[30] M. Hasenbusch, Phys. Rev. B 82, 174433 (2010).

[31] A. Peres and L. E. Ballentine, Quantum Theory: Concepts and Methods (Kluwer Academic Publishers, 1995) pp. 131-135.

[32] C. M. Caves, C. A. Fuchs, and P. Rungta, Found. Phys. Lett. 14, 199 (2001).

[33] S. Hill and W. K. Wootters, Phys. Rev. Lett. 78, 5022 (1997).

[34] H. S. Dhar, A. K. Pal, D. Rakshit,A. Sen(De), and U. Sen, Monogamy of Quantum Correlations- A Review, in Lectures on General Quantum Correlations and their Applications, edited by F. F. Fanchini, D. de O. S. Pinto, and G. Adesso, Quantum Science and Technology Series (Springer, Cham) (2017).

[35] S.-J. Gu, H.-Q. Lin, and Y.-Q. Li, Phys. Rev. A 68 (2003).

[36] A. Montakhab and A. Asadian, Phys. Rev. A 82, 062313 (2010).

[37] M. Kargarian, R. Jafari, and A. Langari, Phys. Rev. A 77, 032346 (2008). 
[38] F.-W. Ma and X.-M. Kong, Phys. Rev. A 84, 042302 (2011).

[39] M. Kargarian, R. Jafari, and A. Langari, Phys. Rev. A 76, 060304(R) (2007).

[40] X.-F. Qian, T. Shi, Y. Li, Z. Song, and C. P. Sun, Phys. Rev. A 72, 012333 (2005).

[41] M. F. Yang, Phys. Rev. A 71, 030302(R) (2005).

[42] M. Hofmann, A. Osterloh, and O. Ghne, Phys. Rev. B 89, 134101 (2014).

[43] L.-H. Shao, Z.-J. Xi, H. Fan, and Y.-M. Li, Phys. Rev. A 91, 042120 (2015).
[44] S. Rana, P. Parashar, and M. Lewenstein, Phys. Rev. A 93, 012110 (2016).

[45] C. Radhakrishnan, M. Parthasarathy, S. Jambulingam, and T. Byrnes, Phys. Rev. Lett. 116, 150504 (2016).

[46] A. P. Majtey, P. W. Lamberti, and D. P. Prato, Phys. Rev. A 72, 052310 (2005).

[47] P. W. Lamberti, A. P. Majtey, A. Borras, M. Casas, and A. Plastino, Phys. Rev. A 77, 052311 (2008).

[48] J. Briët and P. Harremoës, Phys. Rev. A 79, 052311 (2009).

[49] L. Zhang, J.-M. Wang, and Z.-H. Chen, Phys. Lett. A 382, 1516 (2018).

[50] C. Radhakrishnan, I. Ermakov, and T. Byrnes, Phys. Rev. A 96, 012341 (2017). 